\title{
FUNCTIONAL PART FAMILIES AND DESIGN CHANGE FOR MECHANICAL ASSEMBLIES
}

\author{
Madan Mohan Dabbeeru* \\ Center for Robotics \\ Indian Institute of Technology Kanpur \\ Uttarpradesh, Kanpur, India \\ Email: mmadan@iitk.ac.in
}

\author{
Amitabha Mukerjee \\ Computer Science \& Engineering \\ Indian Institute of Technology Kanpur \\ Uttarpradesh, Kanpur, India \\ Email: amit@cse.iitk.ac.in
}

\begin{abstract}
We consider two questions related to functional part families: a) how to characterize function in a computational framework, and b) how does the structure-to-function model generalize when the design changes, e.g. by changing the set of design variables? For the first, we observe that function is defined on the space of behaviours of the part, whereas structure is defined in the space of design parameters. For mechanical assemblies, as the design parameters change, their effect on the motion parameters can be complex, and cannot be automated in full generality. Thus, the mapping from structure-to-function involves considerable designer knowledge. For computational purposes, we quantify this function by defining part-family-specific Configuration Space (C-space) constructions, and also a metric that operates on these $C$-spaces to define each function.

When the design is changed, either by changing the design space (structure), or by the user expectation (function), can existing design knowledge from the earlier part family migrate to the new product family? We make a start towards exploring how this knowledge can be modified when the part family is evolved, for example by introducing additional design variables, or by changing functional roles. Using examples from several lock designs, we present a small prototype for this process of modeling function and design change, implemented on a commercial CAD engine.
\end{abstract}

\footnotetext{
*Address all correspondence to this author.
}

\section{Function in Design Process}

Functional considerations permeate every stage of design, from the earliest conceptual thinking to detailed design optimizations. Yet the concept of function remains unclear in the design community [1]. Certainly, function is related to the design object's behaviour, loosely defined as the totality of the object's interactions with itself and its environment. At other times, it is related to the user's expectations of the part, which highlight certain aspects among the behaviours. In this work we interpret function in terms of user expectations and consider the behaviours as a discrete set $B$, then a subset $F$ that meets the design intent may constitute the "function". For computational work on function, we quantify this function in terms of a performance metric that reflects the degree to which the function meets the user's expectations.

We consider the class of mechanism assemblies, in which the relative motion of different parts is captured through a configuration space (C-space) [2], which is used as an index to retrieve the intended behaviours of the existing mechanisms [3]. The Function-Behavior-Structure (FBS) [4] framework relates the function of a design object to its behaviors and its structural descriptions.

However, functions are not independent of the user. Let us consider the example of a padlock. Initially, its function was seen as that of providing an open-able ring (topological torus). However, once it is instantiated, other aspects of its behaviour such as its weight, or how noisy it is, may become part of the user expectations. Thus, function may be determined by a) mapping 
the structure/geometry into a comprehensive set of behaviours, and b) defining performance metrics on some or all of these behaviours. Both these problems are intractable for general designs, where determining all behaviours is problem-specific and does not generalize at all; similarly defining performance metrics is also usage specific.

One of the important questions is that the nature of the design variable space itself changes as function proceeds. For example, some inter-relations between the variables may prove to be untenable based on functional considerations, so that singularities emerge in the design space. Also, as the functional aspects become clear, the relative importance of different design variables change, and the nature of approximations used in arriving at a mathematical model can be changed. These would also affect the part family structure-to-function mapping computations.

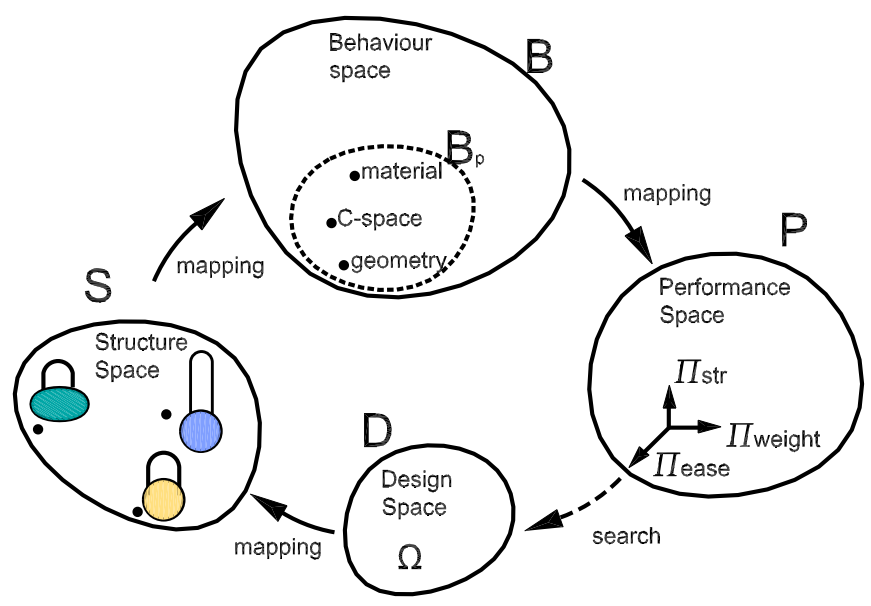

Figure 1. Design Process: A mapping from design space to structure space and searching the design space based on performance evaluation of the intended behaviours.

In this work we consider these problems of identifying function and relating this to design change in the context of functional part families. Initially, a functional part family is defined by a shared structure and a shared function, defined in terms of a set of performance metrics. However, later, either the structure or the function may change. Here we consider one kind of design change where the set of parameters related to a design space is expanded from the existing design space to the new design space by introducing new design parameter into the existing design space and show how the performance metrics would change in functional part families and also we examine how the design constraints vary based on functional constraints.

Initially we identify a set of design parameters in a design space (D) and each design vector $\underline{v}$ in the design space can be mapped to an unique structure in structure space (S). The main aspect here involves identifying the set of intended behaviours $\left(\mathrm{B}_{i}\right)$ in the whole set of behaviours (B), and then defining performance metrics on these intended behaviors in performance space $(\mathrm{P})$. These intended behaviors can also be termed as the performative behaviours $\left(\mathrm{B}_{p}\right)$ since the performance metrics can be defined only on this subset. These performative behaviours are similar to what Gero [5] calls expected behaviours. Thus to re-phrase the F-B-S model of John Gero [5], we may say that our model involves D-S-B-P, which involves mapping from $\mathrm{D}$ to $\mathrm{S}$, $\mathrm{S}$ to $\mathrm{B}$, and then evaluation from $\mathrm{B}$ to $\mathrm{P}$. The results of evaluation are then used to search in design space and come up with a set of improved S. This process is shown in Fig. 1. In this paper we characterize function based on this frame work for functional part families.

\subsection{Function in Part Families}

Part family is a set of parts that serve a related set of market applications - they are functionally similar, and share a common technology base, and lead to better processes for life-cycle design [6]. Functional commonalities across product families have been considered by $[7,8]$, but even here, not much progress has been made in mapping the structural similarities onto function. Here, we distinguish between two types of part similarities: (i) Functional Part Family, which shares an embodiment and (ii) Geometric Part Family, which shares the same geometric structure, differing only in dimensional parameters.

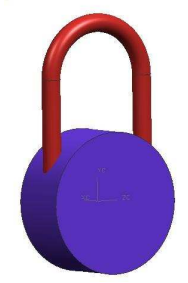

Lock A

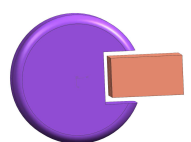

Lock B

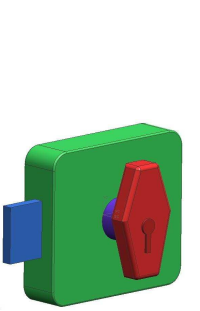

Lock E

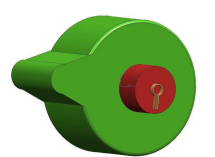

Lock C

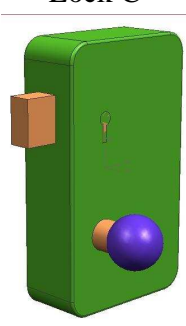

Lock F
Figure 2. Lock Family: Functional part families are evaluated based on the same set of qualities. Though the locks shown are varying in geometry but their shared functions can be locking, strength of the lock, ease of locking etc. 
Definition 1. Functional Part Family (FPF) is a set of members, in which each member share the same qualitative nature and semantics of functions but the specific performance metric may be different since the design vectors can be different.

Definition 2. Geometric Part Family (GPF) is a set of members, in which each member will share the same set of performance measures $\mathcal{P}$ and consequently they also have the same design vector space $\Omega$.

A FPF is that set of related parts where knowledge of function can be transferred in some meaningful way. Since this cannot be defined clearly, we adopt the notion that an FPF is a set of designs that are evaluated based on the same set of qualities; i.e. the actual metrics may be different (since the design spaces or embodiments differ) but the semantics of what is being measured (e.g. strength, ease of locking) remain the same. Hence the primary function of the six locks shown in Fig. 2 is "locking". Within a GPF, different instances arise as a result of variations in a small number of design variables. We show that given any set of design variables one can generate the $\mathrm{C}$-space, and also that the performance metrics can be evaluated on this resulting $\mathrm{C}$-space. Thus we can explore different designs that arise within the constraints defined by the designer.

\subsection{Configuration Space}

Configuration Space (C-space) is the space of independent variables describing relative motions of sub-parts [9]. The Cspace of a kinematic pairs can be defined interms of the shapes and degrees of freedom of its parts. In Fig. 3(c), the hatched region correspond to invalid object positions; the free region correspond to valid object positions; and the boundary separating these two regions is the contact space. Contact space is the trajectory where the two object touch with geometric features vertex,edge and forms feature contacts vertex-vertex, vertex-edge, edge-edge type.

Definition 3. Configuration Space $C$ of a body w.r.t another is the space of all configurations $\vec{u} \in \mathcal{C}$ the bodies can have w.r.t one another. The Obstacle space of body $B$ w.r.t $A, O S_{\mathcal{A}}(\mathcal{B})$ is defined as the set of motions that cause a collision between $A$ and $B . O \mathcal{S}_{\mathcal{A}}(\mathcal{B})=\left\{\vec{u} \mid \exists(x \in A) T_{B}^{A}(\vec{u}) x \in B\right\} . \delta O S_{\mathcal{A}}(\mathcal{B})$ is the boundary of this obstacle space.

Computing the (C-space) for general motions remains an intractable problem [10]. Further, given a C-space, obtaining successful abstractions on it - i.e. segmenting the free-space into behaviourally significant regions -e.g., using topologically different contact types [11], remains a considerable challenge. In the situation involving part families, we assume that the $\mathrm{C}$-space model has been worked out for some existing members in the (a)

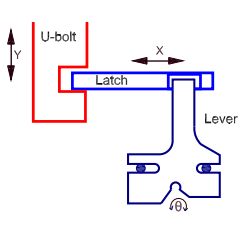

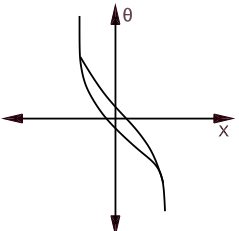

(b)

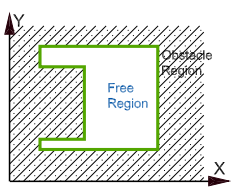

(c)
Figure 3. Configuration Space a) Mechanisms in the lock can be decomposed into a key-barrel fragment and a latch-bolt fragment b) C-space for key-barrel design fragment - rotation $\theta$ of key results in $X$ translation movement in latch. due to the hysteresis loses the $X$ varies as shown.c) C-Space for latch-bolt design fragment - relates horizontal motion of latch $(X)$ with vertical motion of u-bolt $(Y)$.

family, so that these can now be extended to the new part being designed or the existing part being analyzed. Also, the qualitatively important aspects of the C-space are also computable based on similar examples. Thus the problem is considerably simpler for part families. Consider six different padlock designs Fig.2, in which A, B and C exhibit Bolt-Latch design fragment while Fig.3a, relates $X$ to the bolt movement $Y$.

In particular, we make the following claims:

- For mechanical designs involving movable parts, the functionality can be captured through certain operations defined on the $\mathrm{C}$-space.

- It is possible to construct such Configuration Spaces for members of a part family taking into account the variation in dimensions and form (section 2.1).

- Measures of performance, defined in terms of certain behaviours, can be related to metrics defined on the configuration space or other functions of the design variables (section 2.2).

By relating the structure, definable in terms of design variables, to a set of behaviours which are evaluated using the performance metric, one has created the basis for optimizing these variables based on function.

\subsection{Performance Measures}

Definition 4. Performance Measure $\boldsymbol{P}$ is a set of real valued functions from a set of behaviours to $\mathfrak{R}$. A set of performance measures $\pi_{i}$ are defined for the set of behaviours intended for the design.

\section{Example : Padlock}

For the padlock the motion of the U-bolt $(X)$ w.r.t the motion of Latch $(Y)$ defines the C-space shown in Fig.3c. The hatched region corresponds to collision configurations; the white region corresponds to "free space". 


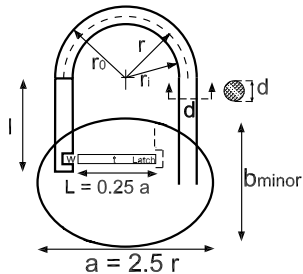

(a)

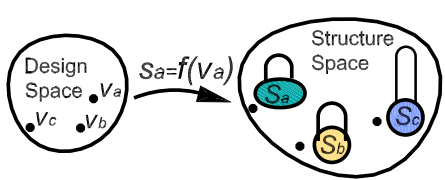

(b)
Figure 4. Design Variables for the Geometric Part Family A:(a) $b_{\text {minor }}$ : height of the elliptical main body; $r$ : U-bolt curvature; $l$ : length of U-bolt. Two other parameters, $w$ and $t$, width of the slot and latch thickness, are not seen here. (b) All other dimensions related to shape and function are determined from these design variables alone, e.g. the width (major axis) of the elliptical body, $a$ is specified as $2.5 r$.

\subsection{C-space Analysis}

Qualitatively, we may think of the latch position Left, Right $(\mathrm{L}, \mathrm{R})$ and the bolt position as Up, Down (U,D) as well as an Intermediate region as (U,I,D). Note that the bolt is in the Up or Down positions, the latch can go Left, but in the Intermediate position(I), neither Up nor Down, the latch can only be Right. This is shown in 5, where we have labelled the regions of the freespace: UL is (Up + Left) etc. In principle, this C-space can be computed for the entire part family. If we consider $T_{L}^{U}$ as the co-ordinate transformation specifying the frame for the $\mathrm{U}$-bolt [U] in terms of the Latch frame [L], then this transformation has two translational DOFs $y$ and $x$. For vertex-vertex type of contact where $\underline{v}_{L}$ on latch is coincident with $\underline{v}_{U}$ on U-bolt. Now we may specify the boundary of the C-space in this extremely simple translation-only system by these equations:

$$
\begin{array}{lc}
\text { vertex-vertex contact: } & T_{L}^{U}(x, y)^{L} \underline{v}_{L}={ }^{U} \underline{v}_{U} \\
\text { vertex-edge contact: } & T_{L}^{U}(x, y)^{L} \underline{v}_{L} \in{ }^{U} \underline{e}_{U} \\
\text { edge-edge contact: } \quad \exists \beta \in T_{L}^{U}(x, y)^{L} \underline{e}_{L}(\beta) \text { s.t } \beta \in{ }^{U} \underline{e}_{U}
\end{array}
$$

Solving these equations for $(X, Y)$ gives us the boundaries of the free-space region in the C-space. When there is an "effort" on Latch $F_{x}$ or on U-bolt $F_{y}$ there will be change in the states by moving in the respective motion axis Table.5. Thus, when the Ubolt is Down (D) and the latch is Left(L), the qualitative configuration is designated DL (Fig. 5a). It is not possible for the U-bolt to move upwards, hence we call this configuration"locked". The act of shifting the configuration horizontally to a point in the DR 6. From here the U-bolt is free to move up to UR. This behavior is systematic across the family, only certain performance criteria, defined on this behavior. Within any member of FPF this will remain a valid characterization of its $\mathrm{C}$-space. However, the degree to which different user expectations are met may vary; these are captured through performance metric.

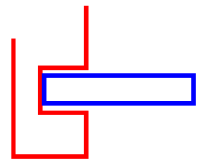

(a)

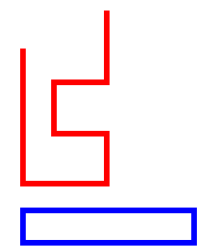

(d)

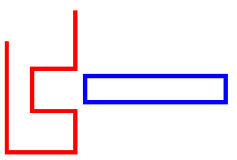

(b)

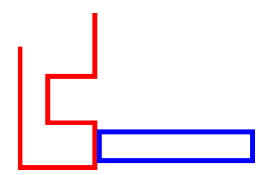

(e)

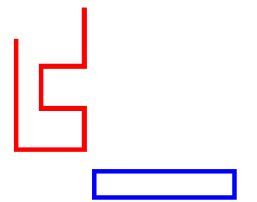

(c)

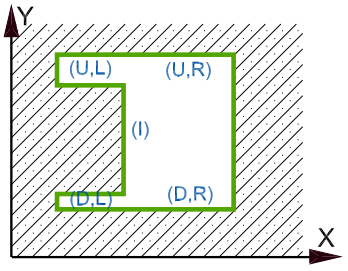

(f)
Figure 5. Functional States as Regions in C-space. The states DL, $\mathrm{DR}, \mathrm{UR}, \mathrm{UL}$ and I are shown in the C-Space (f). Behaviours involve legal transitions between these states. thus, the fact that from $(D, L)$ one can transition only to $(D, R)$ and that this motion involves the use of the key, defines the primary function of the lock.

\begin{tabular}{|c|c|c|c|c|c|}
\hline \multicolumn{3}{|c|}{$(U, L)$} & \multicolumn{3}{|c|}{$(U, R)$} \\
\hline$F_{y}$ & $F_{x}$ & State & $F_{y}$ & $F_{x}$ & State \\
\hline 0 & + & $(U, R)$ & 0 & + & \\
\hline+ & 0 & & + & 0 & \\
\hline 0 & - & & 0 & - & $(U, L)$ \\
\hline- & 0 & & - & 0 & $(D, R)$ \\
\hline \hline \multicolumn{3}{|c|}{$(D, R)$} & \multicolumn{4}{|c|}{$(D, L)$} \\
\hline$F_{y}$ & $F_{x}$ & State & $F_{y}$ & $F_{x}$ & State \\
\hline 0 & + & & 0 & + & $(D, R)$ \\
\hline+ & 0 & & + & 0 & \\
\hline 0 & - & $(D, R)$ & 0 & - & \\
\hline- & 0 & $(U, R)$ & - & 0 & \\
\hline
\end{tabular}

Figure 6. Operation States of Bolt-Latch. Only certain transitions are permitted between different c-space regions. thus, if a positive force is applied along $\mathrm{Y}$ in state $(D, L)$, there is no change of the state. only applying a positive force in $\mathrm{X}$ can move it to state $(D, R)$.the motion states with + , - indicate positive, negative motion.

\subsection{Performance Metrics}

The product performance can be defined as the how well the product implements its functional elements. Let us consider functions that it is desirable that a padlock must perform. As an initial point, we may define

- $f_{0}$ : Create a topological torus (ring) structure which can be opened or closed.

In order to meet this function, a design with a key mechanism and a latch entering into a slot on a shackle is designed. This corresponds to decomposing $f_{0}$ into two sub-functions: 
This function could be decomposed as

- $f_{\text {linear }}$ : Operation of key in rotary motion converted to linear motion to move the latch.

- $f_{\text {ring }}$ : Ring closes when latch is moved into slot with shackle in "down" position, and opens when it is withdrawn. (Design fragment implemented in the C-space of Fig. 3b)

$f_{\text {ring }}=\{\exists x \mid$ PreventsTrans $(y)\}$

All members in the geometric part family share functions $f_{\text {linear }}$ and $f_{\text {ring }}$ which are key function in this GPF. For all members of this $\mathbf{P F}$, the $\mathrm{C}$-space must implement the functions $f_{\text {linear }}$ and $f_{\text {ring }}$ (and thereby $f_{0}$ ). However additional functions may be important design considerations for different members of the part family. For example, some users may require extremely high strength in their locks (maximize the breaking force) strength of lock $\left(\pi_{s t r}\right)$, while others may require that they should be openable and lockable easily (for example in the dark). Other performance measures ease of locking $\left(\pi_{\text {ease }}\right)$, weight of lock $\left(\pi_{\text {weight }}\right)$ are some of the design considerations for the part family explained in the following sections.

2.2.1 Ease of Locking Consider the space of the design variables $w, t$ Fig.7. Clearly, if $w<t$, then the function of locking fails. If $w-t$ is small, user may find it difficult to guide the latch into the slot on the U-bolt. Thus a simple measure for ease of locking is just the clearance $w-t$, which should be maximized subject to other design constraints. The performance metric for ease of locking is

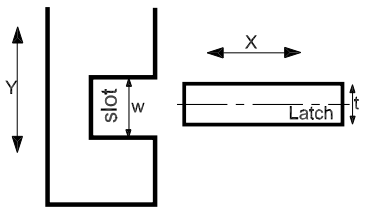

(a)

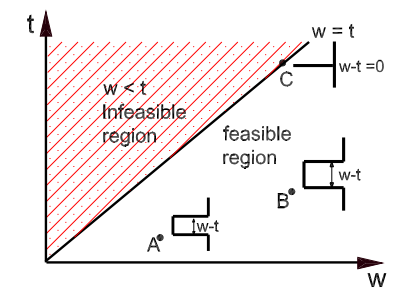

(b)
Figure 7. Variation in C-space within Geometric Part Family.(a)Latch-Bolt design fragment - relates horizontal motion of latch $(X)$ with vertical motion of u-bolt $(Y)$ (b) AS $w-t$ reduces, the clearance for moving the latch reduces. if this gap is too small, ease of locking may be hindered by small misalignments; if it is too large, it may leave too much space for the latch to bend in. the slot part of three different designs are shown in A, B, C.

The performance metric for ease of locking is thus defined as

$$
\pi_{\text {ease }}=w-t
$$

which is to be maximized. Any other signed monotonic function $\pi_{\text {ease }}=(w-t)^{3}$ could also be used.

2.2.2 Strength of the Lock The strength of the lock depends on a number of factors, such as the tensile strength of the U-bolt, the bending strength of the latch, the groove on the U-bolt, the support for the latch inside the lock, etc. Here we have considered it as supporting as shown in Fig.8. When the lock is hammered, an impact force is applied near the left end of the latch. While the effect of this impact loading is more difficult to model, a reasonable simplifying assumption is that a lock which is strong in normal loading would also be strong in impact loading. Thus, we seek to maximize the failure force $F$, which may be computed by setting the bending stress equal to the yield strength. The stress is expressed as $\sigma=\frac{6 F l}{b_{L} t^{2}}$, and setting this equal to the yield strength gives us a value for $F$ which is to be maximized :

$$
\pi_{s t r}=\frac{\sigma_{Y} b_{L} t^{2}}{6 l_{l}}
$$

where $\sigma_{Y}$ is the yield strength of the material (incorporating a suitable factor of safety etc) and 1 the supporting length.
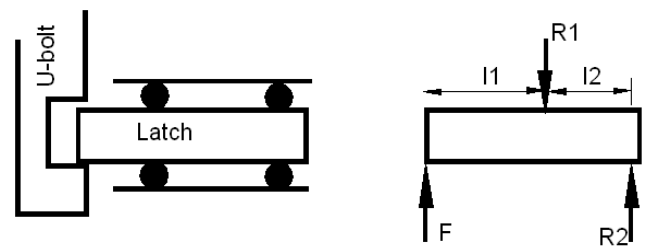

Figure 8. Strength of the Lock. We use a very simple roller support model for the latch.

2.2.3 Weight of the Lock The total weight of the body will be the sum of (a) Weight of the U-bolt, $W t_{u}=$ $\left.\rho_{u}\left(2 l_{\text {bolt }}+\pi r\right) \pi d^{2} / 4\right)$ (b) Weight of the lock body, $W t_{b}=\rho_{b} \pi a b t_{b}$

$$
\pi_{w e i g h t}=W t_{u}+W t_{b}
$$

Here $t_{b}$ is the depth of the lock, and density $\rho_{b}$ reflects the average density of the body, including the empty spaces etc. This gives us the performance measure, $\pi_{\text {weight }}$ to be minimized. 


\subsection{Design Search: Padlock Optimization}

Design is a search process. During this process designers tries to find optimum solutions through searching the design space. The member of the part family is characterized by a set of design variables. In which we focus on a 5-tuple design vector $w, t, r, b_{\text {minor }}, l$ which we call as driving variables as the other design dimensions internal to the lock Fig.4 are defined in terms of these driving variables. For example, the depth of the body is $4 t$ and the width of the latch is $b_{l}=3 t$. Given a set of values for a design vector, one can determine its shape and also various auxiliary components such as the C-space.

\subsubsection{Multi-Objective Optimization}

$$
\begin{array}{lll}
\text { Maximize } & \pi_{s t r}(\underline{v})=\frac{\sigma_{Y} b_{L} t^{2}}{6 l_{l}}, \\
\text { Maximize } & \pi_{\text {ease }}(\underline{v})=w-t, \\
\text { Minimize } & \pi_{\text {weight }}(\underline{v})=W t_{u}+W t_{b}, \\
\text { Subject to } & g(\underline{v}) \equiv w-t \geq 0.1, & 4.0<w, t<8.0 \\
& 12.0<b_{\text {major }}<25.0, & 10.0<r, l<20.0
\end{array}
$$

We have used Evolutionary Multi Objective (EMO) algorithms (NSGA-II) [12]. The probabilities of recombination and mutation operators used are $p_{c}=0.8$ and $p_{m}=0.3$ respectively. The Fig. 9 shows the Pareto front. Considering the three objective functions ease of locking, strength of lock, and weight of lock we have obtained a set of optimal solutions, non-dominated solutions, Pareto front. Pareto optimal front aids the decision maker to chose the non-dominated solution. Any point in Fig.9 gives the respective design vector which defines the shape of the the design object with desired function. In each generation, different design vectors generate various design objects by satisfying the function. Fig. 10 shows locks in various generations.

\subsection{Resulting Designs}

In this section we are going to discuss the evaluation of performance measures on the intended behaviours. The intended behaviors we consider are $\pi_{\text {ease }}, \pi_{\text {str }}, \pi_{\text {weigth }}$ and the multi-objective optimization procedure NSGA-II is used. Fig.9(a) shows the Pareto optimal solutions for different weights $\mathrm{w}=0.4, \mathrm{w}=0.8$ etc and the Fig.9(b) shows the Pareto optimal solutions for different ease of locking and Fig.9(c) shows the Pareto optimal solutions for the different strength values.And Fig.10 shows the locks in different generations.

\section{Design Fragment : Slotted Wheel and Latch Mech- anism}

In this section we consider a design fragment (Fig. 11(a)), in which slotted wheel has rotational DOF $\theta$ and the latch has
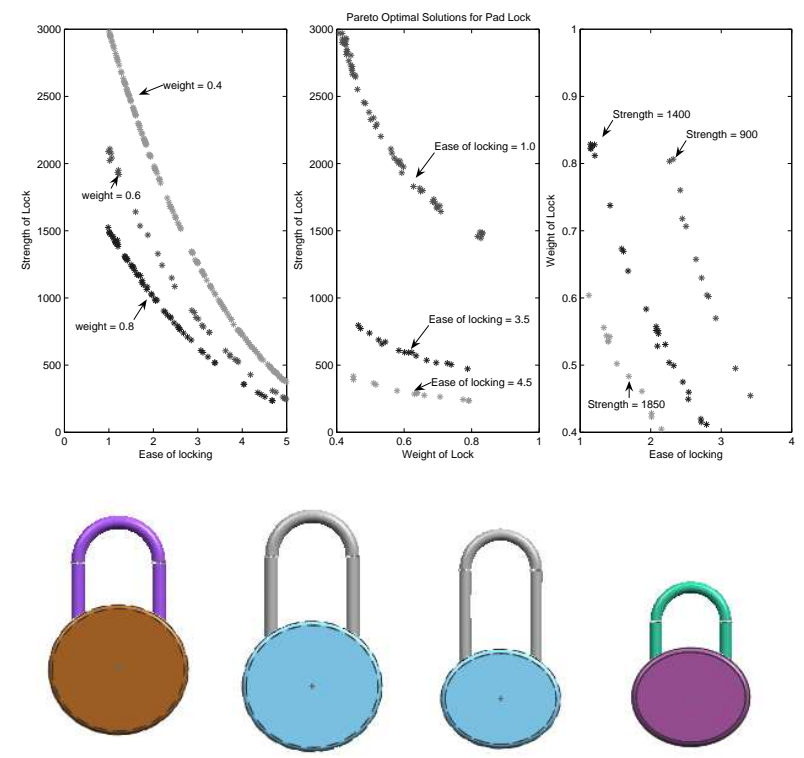

Figure 9. Pareto Optimal Solutions Pareto fronts with all nondominated solutions are generated in every generation during the design optimization process. the designer can explore the possible nondominated designs and decide on a tradeoff between several design objectives.
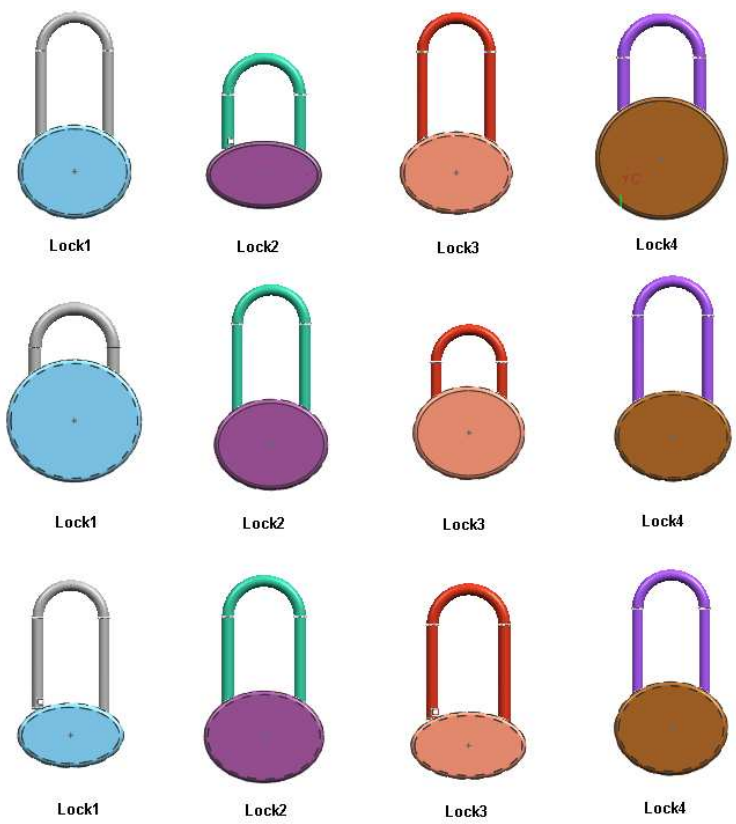

Lock3

Lock4

Lock1

Lock2
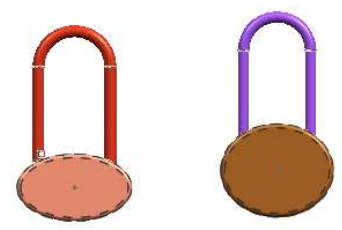

Lock4

Figure 10. Geometric Part Family : Locks in various generations

translational DOF $X$. The primary function of this mechanism can be, 


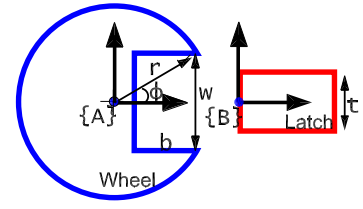

(a)

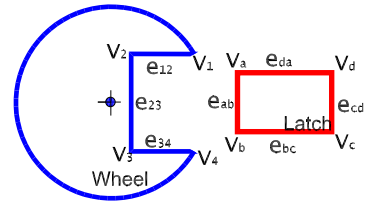

(b)
Figure 11. Slotted-wheel mechanism. (a)Design parameters for slotted-wheel mechanism: radius $r$, width $w$, breadth $b$, thickness of the latch $t$ and $\phi=\sin ^{-1}\left(\frac{w}{2 r}\right)$. $\phi$ is a dependent parameter on design parameters. (b) Its geometric elements' representation.
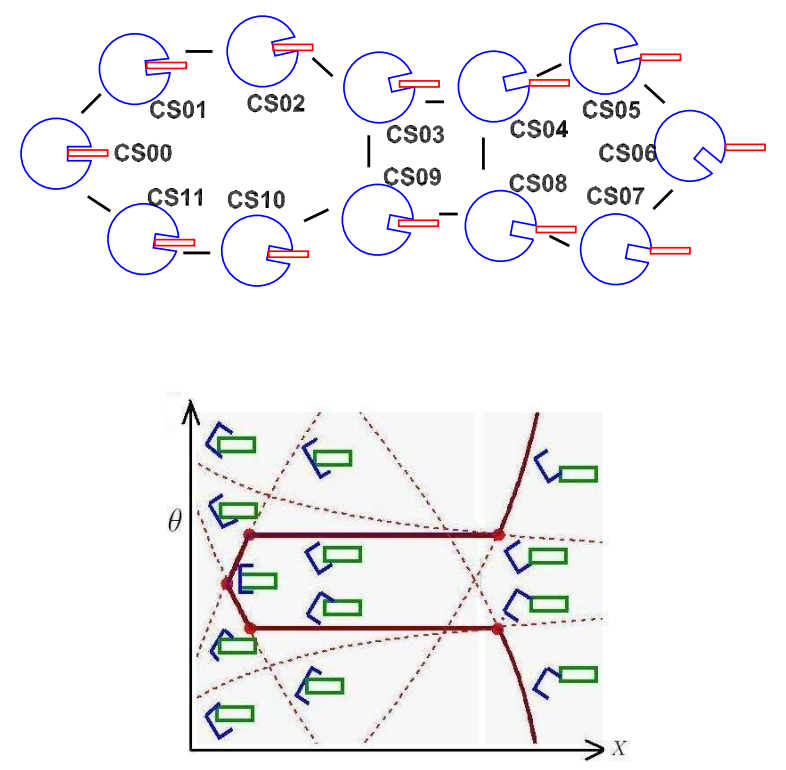

Figure 12. (a) Contact State Graph: Each node represents a contact state and the edge represents the transition of contact states. the contact state transition is specified a priori as in general case there are many possible contact states possible in this planar case. by considering only subset of possible contact states chosen based on the heuristics for successful assembly to achieve the desired function $(b)$ contact curves represent the motion behaviours of the parts in different contact states.

- $f_{0}$ : The horizontal movement of the latch into the rotating slotted wheel locks the rotational movement of the wheel.

The function of this mechanism can be captured by the motion behaviour and the type of contacts among the topological contacting surface elements (vertex, edge). Fig. 11(a) shows the contact space and 11(b) shows the configuration space of the mechanism. Any device which shares this function may have to satisfy the behaviours represented through C-space (Fig.11 (b)). With the help of this mechanism we explain how the design change may affect structure-to-function model in functional part families in the next section.

\section{Design Change}

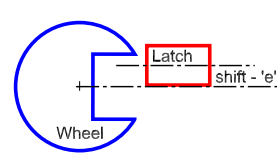

(a)

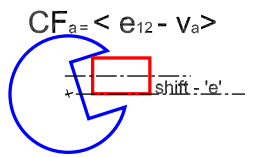

(b)

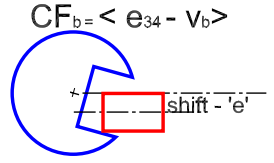

(c)
Figure 13. Vertical Shift in Latch (a) Note that in addition to the contact formations of Fig. 12 two new contact formations are observed for shifts that are positive $\left(C F_{a}\right.$,Fig.(b)), or negative $\left(C F_{b}\right.$, Fig.(c)).

A key process during any design interaction is the redefinition of the design space itself. Expert designers often question the design specifications more insightfully than novices [13]. Computationally, the important question is if the models formed for a particular design space can be incorporated into new conceptual structures. Design change may involve redefinition of constraints defined in the early stages - in general, the design problem defined in the early stages tends to be under-constrained [14]. As the design process progresses a new set of constraints arise based on the functional specifications. As a very simple example, the constraint $(w>t)$ used in Eq. 4 for the class of padlocks is easy to discover but the $(w, t)$ space in the rotating barrel lock (Fig. 11) will experience different feasible and infeasible regions based on functional constraints.

In this section we examine how the design change may affect the structure-to-function mapping in functional part families. For this, we consider the rotating barrel lock (Fig. 11) and examine how the change in motion behaviors affect the performance metrics in functional part families, with the incorporation of a new design variable.

\subsection{New Design Variable: Vertical Shift $e$}

Consider now a slotted-wheel lock mechanism with a latch whose translational axis is displaced from the slotted wheel center by a distance $e$ (Fig. 13a). The mechanism is in the locked state when the latch is inserted into the slot of the rotating wheel. Clearly, as $e$ becomes more than $\frac{w-t}{2}$, the degree of penetration would vary, and this would affect its function. Here, the strength of the lock may be measured in terms of the maximum 
torque that the barrel can withstand, instead of the vertical force. In case of positive vertical shift $e$, the latch can enter into the slot only between $\theta_{1}$ and $\theta_{2}$ where $\theta_{1}=\sin ^{-1}\left(\left(\frac{t+2 e}{2 r}\right)\right)-\phi$ and $\theta_{2}=\phi-\sin ^{-1}\left(\frac{t-2 e}{2 r}\right)$. We consider the vertical shift as a design change and introduce $e$ as one of design parameters in the design space $\Omega$, hence the design vector $v$ will be $\{r, w, t, b, e\}$. With this design change we find out the feasible and infeasible regions in the design space $(w, t)$ based on the functional constraints.

With the presence of vertical shift $e$ in the fixed axis of the latch (Figure 13(a)), new contact formations can be possible (Figure 13(b),(c)). These new contact formations may characterize the new behavior of the mechanism and hence the performance metrics. For example, the penetration depth varies with the variation of shift $e$ values as shown in the Fig. 14b, c and d. In the next subsection 4.2.1 we investigate the maximum penetration depth for different shift values and hence we will discover the feasible and infeasible regions in the design subspace $(w, t)$ for the functional part family design optimization.

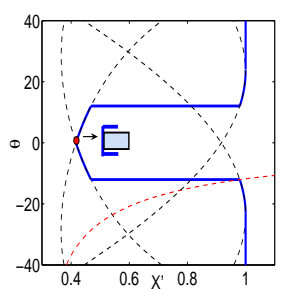

(a)

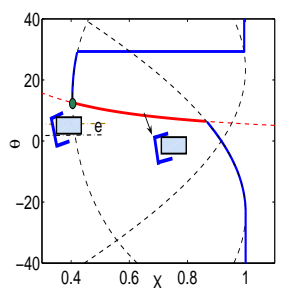

(c)

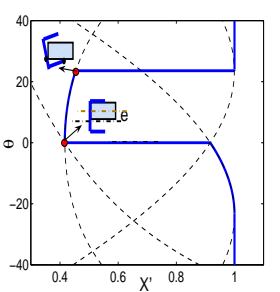

(b)

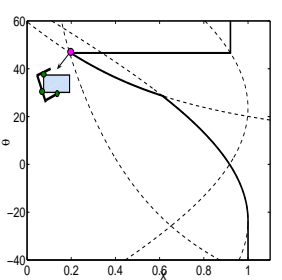

(d)
Figure 14. (a)C-space without any axial shift, and the maximum penetration is possible at $\theta=0(\mathrm{~b}) \mathrm{C}$-space with an axial shift $e=\frac{(w-t)}{2}$. (c) C-space where new contact state $C S_{a}=\left\langle e_{1}, v_{a}\right\rangle$ is possible and hence the penetration depth varies with the rotation of wheel. (d) C-space with $C F_{c}=\left\{P C_{1}, P C_{2}, P C_{3}\right\}$ at a maximum penetration. $P C_{1}$ is $C S_{a}$, $P_{2}$ is $C S 03$ and $P C_{3}$ is $C S O 1$

\subsection{Performance Metrics}

4.2.1 Maximum Penetration The penetration depth (PD) varies with the change in the vertical shift $e$ values shown in Fig 15 . With the presence of shift $e$ values, the contact formations are also varying where the maximum penetration is possible.

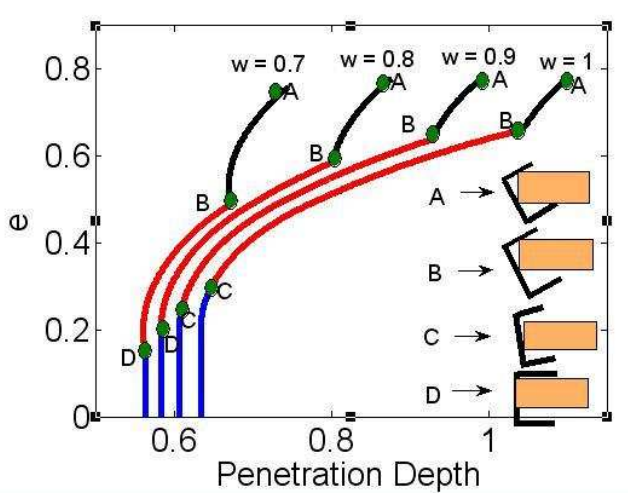

Figure 15. Maximum penetration depth for different ranges of axial shifts. $\mathbf{A}, \mathbf{B}, \mathbf{C}$ and $\mathbf{D}$ are the different contact states at which maximum penetration is possible for a design variable. $\frac{t}{r}: 0.4, \frac{b}{r}: 0.5$

Based on this computation, we can define a performance metric $\pi_{P D}=$ penetration-depth. However, specifying the penetration depth alone may not be adequate, since for high values of $e$, if this maximum penetration occurs at values of $\theta$ of $45 \mathrm{de}-$ grees or so, it may not be effective in preventing lock rotation. Thus, we may also wish to specify the maximum $\theta$ at which this rotation occurs. The the $(w, t)$ design subspace, with feasible (FFR) and infeasible regions, are shown for differing values of $\pi_{P D}$ along with various constraints on $\theta$, in Fig.17(a) and (b). For example, the second image considers the case where where $\pi_{P D}>=0.54$, at angle $\theta<18^{\circ}$ and Fig. 17(a) shows the situation without any minimum $\theta$ constraint for a different $\left(\pi_{P D}\right)$.
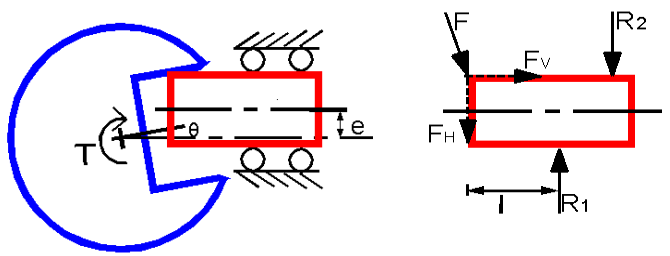

Figure 16. Strength Analysis: A typical contact configuration; here the maximum contact force is determined given the maximum torque that the slotted wheel axis is expected to bear. this maximum torque $\tau_{\max }$ is equal to the contact force times a moment arm $d(X, \theta)$ that depends on the penetration depth of the latch. 


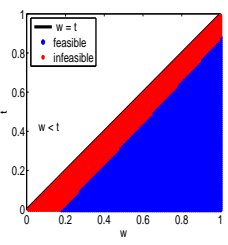

PD 0.5 and any $\theta$

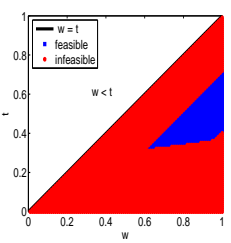

PD 0.54 and $\theta<18^{\circ}$

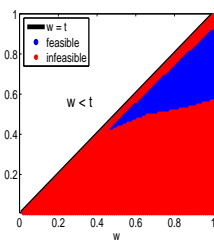

$\tau>6$
Figure 17. Evolving Constraints Based on Performance Measures $\pi_{P D}$ and $\pi_{s t r}$. (a),(b) Feasible regions (FFRs) in the $w, t$ design subspace for differing constraints on $\pi_{P D}$ or the maximum penetration, together with constraints on $\theta$. the last figure shows FFRs for constraints on $\pi_{s t r}$ or the maximum torque $\left(\tau_{\max }\right)$. (slotted wheel mechanism, axial shift $e=0.3$ and $b=0.5$ )

4.2.2 Strength: Maximum Torque If we are to evaluate this lock based on its strength, an obvious measure would be the maximum torque $\tau_{\max }$ it can support. This torque will vary in different contact states, and we may compute it for the maximum penetration, as discussed in section 4.2.1. Let us consider a contact state $\mathbf{B}$ as shown in Fig. 15. At this contact state the latch in the mechanism is considered as a simple supported, for which the free body diagram is shown in Fig. 16. Also, Eq. 5 gives the relation for the maximum strength of the latch which can withstand against the desired torque $\tau$.

Here we obtain the following constraints based on Fig. 16. The maximum torque $\tau_{\max }$ that can be supported is determined by the contact force $F$ and its moment arm; this contact force is in turn limited by the latch strength.

$$
\begin{aligned}
F & =\frac{\sigma_{Y} b_{\text {latch }}\left(t_{1}\right)^{2}}{6 l} \\
\tau_{\max } & <-F \cos \theta d(X, \theta)-F \sin \theta\left(\frac{e}{r}+\frac{t}{2 r}\right) \\
d(X, \theta) & =\frac{2 e \cos \theta+t \cos \theta-w}{2 r \sin \theta}
\end{aligned}
$$

where $d(X, \theta)$ is the moment arm for the vertical component of the contact force $F$. The other terms are as in Eq. 2 for the padlock.

The performance metric $\pi_{s t r}$ for the slotted wheel may be simply set to be $\tau_{\max }$ as given in the equation (Eq. 5. Now, asserting different levels of acceptability for $\pi_{s t r}$ results in constraints involving different parts of the design space, as seen in Fig. $17(\mathrm{c})$. Designs with thin latches (low values of $t$ )are clearly rejected by the requirement for strength, but owing to the penetration depth being related to theta, when $e$ is a significant compared to $t$, high strength also requires higher slot width $w$ 's.

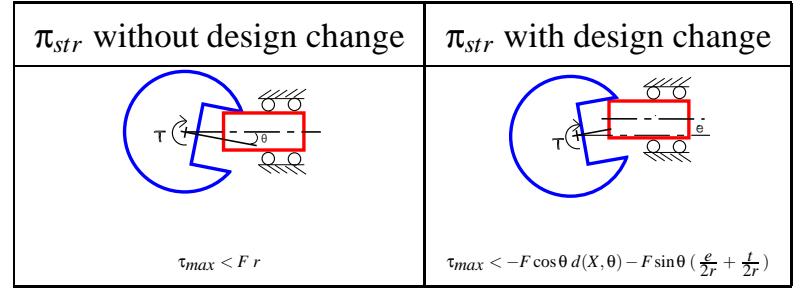

Table 1. Performance metrics with and without design changes considered in rotating barrel lock.

And Table 1 shows the performance metrics considered with and without design change in rotating barrel lock.

With this, one may learn some patterns for those regions of the design space that are more likely to contain the functionally feasible part families. The discovered feasible regions can be added to the design optimization as design constraints for tighter bounds on the design. By using general purpose function approximators such as neural networks one can enable the system to infer feasibility regions from a small subset of part families that are anyhow being explored in another design task, which is currently under investigation.

\section{Conclusion}

There are two primary contributions in this work, to present a workable notion of function in design for part families, and to consider (in a preliminary manner) how this notion of function can be expanded under certain design change situations. Design change is a common theme of human design, yet machines are limited to search only within the given specifications. We explore only one kind of design change, and show how the designer's knowledge of function in an earlier space can be used in a new design space which is a generalization of the earlier situation.

However, since function depends on the user's perception of the part, it is possible to define function only in terms of the ways in which they are similar or dissimilar to related parts, and not independently of them. By taking the part family as a set of "related parts" this paper shows how such functional models may be developed based on the well-studied notion of Configuration space, and then based on this premise, we have presented an approach for mapping between structure and function within a functional part family. We bring together existing concepts of qualitative decomposition and of configuration spaces to create simple models that can work with variational part families. The resulting designs enable us to consider functions and even evaluate the design in terms of function during the early part of the design process. In our interactions and demonstrations of this system to working designers, initially, experienced designers did not like it since felt that this approach is unlikely to benefit them, because they are already able to bring together considerable diversity to the design task. However, it was felt that for beginning 
designers, the availability of visualizations that present a number of choices in the design space provides opportunities for considering alternatives that may not have been initially obvious. This, we feel, is a main avenue for expanding present CAD systems, where more tools are made available so that less experienced designers can also benefit. In the end however, even mature designers found that some of the ideas thrown up by the system may be useful in promoting creative thought.

The approach to design change is clearly preliminary, based as it is on the extension of the design variables by introducing a new variable, which earlier was assumed to have a fixed value. While the result of this change has been analyzed, clearly there is much work remaining in terms of other forms of design change, e.g., by extending the constraints on the variables, by changing the inter-relationships between the variables, by altering the mapping from the design variables to the geometric variables, and other aspects. In the design change considered here either the design space is changed, or the set of functions may be changed (altering the dimensionality of the Pareto frontier). However, the expected behaviour may deviate from the prototypical family functionality in many ways, and for each of these it would be necessary to manually re-define this new functional need in terms of a performance metric. However, this is a problem that may not be modeled computationally very easily.

Another aspect for future work considers the issue of manufacturing tolerances. Tolerances add new dimensionalities to the space of motions, resulting in higher dimensional configuration spaces, and result in functional ramifications that could not be foreseen in the earlier, canonical C-spaces. Incorporating performance measures that deal with tolerances is equivalent then to dealing with designs that have added design parameters. This is an aspect we are currently exploring.

\section{REFERENCES}

[1] Vermaas, P. E., and Dorst, K., 2007. "On the conceptual framework of john gero's fbs-model and the prescriptive aims of design methodology". Design Studies, 28(2), March, pp. 133-157.

[2] Joskowicz, L., and Sacks, E., 1994. "Configuration space computation for mechanism design". In Proceedings of IEEE International Conference on Robotics and Automation, Vol. 2, pp. 1080-1087.

[3] Murakami, T., and Nakajima, N., 1997. "Mechanism concept retrieval using configuration space". Research in Engineering Design, 9(2), June, pp. 99-111.

[4] Gero, J. S., 1990. "Design prototypes: A knowledge representation scheme for design". AI Magazine, 4(11), winter, pp. 26-36.

[5] Gero, J. S., and Kannengiesser, U., 2002. "The situated function - behaviour - structure framework". Gero (ed.) Artificial Intelligence in Design, pp. 89-104.
[6] Simpson, T. W., 2004. "Product platform design and customization: Status and promise". Artificial Intelligence for Engineering Design, Analysis and Manufacturing, 18, pp. 3-20.

[7] Thevenot, H. J., and Simpson, T. W., 2004. "A comparision of commonality indices for product family design". In ASME 2004 International Design Engineering Technical Conferences and Computers and Information in Engineering Conference, Proceedings of the DETC.

[8] Jiao, J., Simpson, T. W., and Siddique, Z., 2006. "Product family design and platform-based product development: A state-of-the-art review". Journal of Intelligent Manufacturing.

[9] Latombe, J. C., 1991. Robot Motion Planning, first ed. Kluwer Academic Publishers,Boston.

[10] Ji, X., and Xiao, J., 2001. "Planning motion compliant to complex contact states". International Journal of Robotics Research, 20(6), June, pp. 446-465.

[11] Mukerjee, A., and Bhatia, 1995. "A qualitative discretization for two-body contacts". In Proc. of the 14th IJCAI, Vol. 1, pp. 915-921.

[12] Deb, K., 2001. Multi-Objective imization using Evolutionary Algorithms, 1 ed. Chichester, John Wiley and Sons, Ltd.

[13] Ahmed, S., Wallace, K. M., and Blessing, L. T., 2003. "Understanding the differences between how novice and experienced designers approach design tasks". Research in Engineering Design, 14(1), February, pp. 1-11.

[14] Gross, M. D., 1986. "Design as exploring constraints". PhD thesis, Department of Architecture ,Massachusetts Institute of Technology, February. 